\title{
ICE VOLUME CHARACTERIZATION USING LONG-WAVELENGTH AIRBORNE POLSAR DATA
}

\author{
Giuseppe Parrella ${ }^{1}$, Noora Al-Kahachi ${ }^{1}$, Thomas Jagdhuber ${ }^{1}$, Irena Hajnsek ${ }^{1,2}$ and \\ Konstantinos P. Papathanassiou ${ }^{l}$ \\ ${ }^{1}$ German Aerospace Center - Microwaves and Radar Institute, Germany \\ ${ }^{2}$ ETH Zurich - Institute of Environmental Engineering, Switzerland
}

\begin{abstract}
In recent years an increased interest arose in using synthetic aperture radar (SAR) to study and monitor land ice for glaciological applications and climate change research. At long wavelengths, SAR systems can penetrate into glacier ice for several tens to hundreds of meters. This makes them sensitive to near-surface as well as deeper ice volume features. This paper investigates the performance of a volume scattering component, in the course of an electromagnetic (e.m.) model development, to relate Polarimetric SAR (PolSAR) signatures to glacier facie. Indeed, PolSAR ice signatures are still poorly understood, including the importance of scattering from the glacier ice volume and its dependency on frequency and incidence angle. A comparison is performed with airborne Pol-SAR data at L- and P-band collected by DLR's E-SAR system over the Austfonna ice cap in Svalbard, Norway, within the ICESAR campaign.
\end{abstract}

Index Terms - Polarimetric SAR, volume scattering, co-polarization phase, land ice, polar ice.

\section{INTRODUCTION}

In the last decades, the use of airborne and satellite remote sensing techniques has deeply impacted glaciology by significant improvements in scale as well as temporal and spatial resolution of cryospheric observations. Despite the significant progress in mapping spatial extent and surface features of glaciers, large uncertainties remain in estimating reliably glacier accumulation/discharge rates, ice thickness and subsurface/volume structures. The use of SAR techniques has probably triggered the greatest advance for these applications due to its penetration capabilities into the ice volume. Nevertheless, today it is still a major challenge to grasp the structure of glacier ice with SAR remote sensing. In recent years a great attention has been given to model-based decompositions of PolSAR data of glaciers. A first model was proposed in [1] as extension of conventional 3-component polarimetric decomposition models (e.g. [2]).
It assumed a shallow snow-ice interface, together with an oriented sastrugi field on the glacier surface, and an underlying ice volume (of oriented dipoles). This approach was able to interpret many effects in the experimental data, but not all, especially concerning co-polarization phase differences. In this paper the authors attempt to provide a detailed interpretation of experimental data in terms of polarimetric characterization of glacier ice by means of an oriented volume scattering component.

\section{PARTICLE VOLUME SCATTERING}

The approach proposed in this work attempts to explain PolSAR signatures of glacier ice with a volume scattering component from a cloud of oriented particles, in particular, air and atmospheric gases inclusions [3], typically present in ice volumes. Consequently, air and gas bubbles are modeled as a cloud of oblate spheroidal particles mainly horizontally oriented. The scattering from a spheroid is approximated with the superposition of the scattering from three orthogonal dipoles [4] as shown in Fig.1. Hence, the elements of a particle scattering matrix [S] (see eq. 1) can be written as:

$$
\begin{aligned}
& S_{h h}=\rho_{1} \cos ^{2} \tau \cos ^{2} \varphi+\rho_{2} \sin ^{2} \varphi+\rho_{3} \sin ^{2} \tau \cos ^{2} \varphi \\
& S_{v v}=\rho_{1}(\cos \tau \sin \varphi \cos \vartheta+\sin \tau \sin \vartheta)^{2}+ \\
& \quad+\rho_{2} \cos ^{2} \varphi \cos ^{2} \vartheta+\rho_{3}(\sin \tau \sin \varphi \cos \vartheta-\cos \tau \sin \vartheta)^{2} \\
& S_{h v}=\rho_{1}\left(\cos ^{2} \tau \cos \varphi \sin \varphi \cos \vartheta+\cos \tau \sin \tau \cos \varphi \sin \vartheta\right)+ \\
& -\rho_{2} \cos \varphi \sin \varphi \cos \vartheta+ \\
& +\rho_{3}\left(\sin ^{2} \tau \cos \varphi \sin \varphi \cos \vartheta-\cos \tau \sin \tau \cos \varphi \sin \vartheta\right)
\end{aligned}
$$

where $\rho_{1}, \rho_{2}, \rho_{3}$ are the particle polarizabilities, $\tau$ the tilt angle and $\varphi$ the canting angle; $x_{1}, x_{2}$ and $x_{3}$ are the lengths of the three dipoles approximating the particle, and $\theta$ is the look angle. In the case of an oblate spheroid, $x_{2}=x_{3}$ (then also $\rho_{2}=\rho_{3}$ ) and $x_{1}<x_{2}$ The upper part of Fig. 1 shows the radar geometry, where $\underline{k}$ is the propagation vector of the e.m. wave, whereas $\underline{h}$ and $\underline{v}$ represent the wave component in the horizontal and vertical polarization, respectively. 


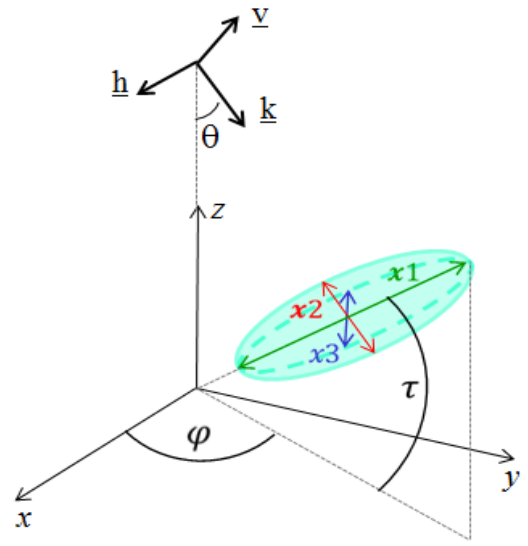

Fig.1: Position of spheroidal particle in its local reference frame $(x, y, z)$ and link to the radar reference frame $(h, k, v)$.

The ratio $\rho_{1} / \rho_{2}$ is defined as particle anisotropy $\left(A_{p}\right)$ and it is related to the particle shape [4]. For $0<A_{p}<1$ we have oblate particles; if $A_{p}=1$ spheres are obtained, whereas for $A_{p}>1$ we have prolate particles (needles for $A_{p} \rightarrow \infty$ ). Moving from a single particle to a cloud, we assume that all the spheroids have same size and are distributed around the canting and tilt angle. In particular uniform distributions are considered with the following angular pdfs:

$$
\begin{aligned}
& p_{1}(\tau)=\frac{\cos \tau}{\sin \tau_{2}-\sin \tau_{1}} \text { for } \tau_{1}<\tau<\tau_{2} \text { and } 0 \text { elsewhere (2a) } \\
& p_{2}(\varphi)=\frac{1}{\varphi_{2}-\varphi_{1}} \text { for } \varphi_{1}<\varphi<\varphi_{2} \quad \text { and } 0 \text { elsewhere (2b). }
\end{aligned}
$$

In this way, a variety of volume cases, from random to absolutely oriented, can be modeled. To account for the superposition of scattering responses from all particles within the cloud, the polarimetric covariance matrix $[C]$ can be calculated, by averaging over the tilt and canting angle distributions as follows:

$$
\begin{aligned}
& {[C]=\int_{\varphi_{1}}^{\varphi_{2} \tau_{1}} \int_{\tau_{2}} \vec{k}_{L}(\tau, \varphi) \cdot \vec{k}_{L}^{*}(\tau, \varphi) p_{1}(\tau) p_{2}(\varphi) d \tau d \varphi} \\
& \text { where } \vec{k}_{L}=\left[\begin{array}{lll}
S_{h h} & 2 S_{h v} & S_{v v}
\end{array}\right]^{T}
\end{aligned}
$$

$k_{L}$ represents the lexicographic scattering vector [4]. As required for dielectric media such as ice, transmission effects at the air/ice interface must be accounted. Transmittivities for $H$ and $V$ polarization are here approximated by the Fresnel formulation [5] and applied on the $[C]$ matrix. In the case of an oriented volume, the model must also account for the anisotropic nature of the ice medium, leading to differential propagation velocities and losses of the e.m. wave along different polarizations [4]. This effect is modeled adapting the approach proposed in [6] to spheroidal particles. The anisotropy of the volume results in different refraction indices for the $H$ and $V$ polarizations (see Eq. 4).

$$
\begin{gathered}
n_{h h, v v}=n_{h h, v v}^{\prime}-j n^{\prime \prime}{ }_{h h, v v} \\
n_{h h, v v}^{\prime}=1+2 \pi N k^{-3} \operatorname{Im}\left\{S_{h h, v v}\right\} \\
n^{\prime \prime}{ }_{h h, v v}=2 \pi N k^{-3} \operatorname{Re}\left\{S_{h h, v v}\right\}
\end{gathered}
$$

In Eq. (4a), $n_{h h, v v}$ is the obtained relative refraction index (normalized to $n_{\text {pure ice) }}$ in the $H H(V V)$ channel and $n_{h h, v v}$ and $n$ "' $h h, v v$ represent its real and imaginary part, respectively. In Eq. (4b) and (4c), $N$ is the number of particles per volume unit, $k$ the wavenumber and $S_{h h, v v}$ the amplitude function of the cloud of particles for Rayleigh scattering, depending on the particle $[S]$ matrix and the permittivity of the particles (air) and the surrounding medium (ice). As [C] changes with distance into the volume due to differential propagation, the total coherency matrix of the volume is the power-weighted average of the changing [C] matrices along depth [7]. In this way, the inclusion of differential propagation leads to a significant increase of entropy and scattering alpha angle from the volume scattering component.

The complete model depends on few parameters: Particle anisotropy $A_{p}$, mean canting and tilt angles $\tau, \varphi$ and their respective distribution width, particles permittivity $\left(\varepsilon_{r}\right)$ and volume fraction $\mu_{i}\left(N=\mu_{i} / V_{\text {sphere }}\right)$.

\section{EXPERIMENTAL DATA}

The data set selected for this investigation was acquired during the ICESAR campaign conducted in March 2007 on the Austfonna ice cap $\left(79-80^{\circ} \mathrm{N}, 20-27^{\circ} \mathrm{E}\right)$, Svalbard archipelago, Norway [8]. This dome-shaped ice cap on the island of Nordaustlandet has a subpolar/polythermal regime and horizontal velocities close to zero [1].

Within the campaign, fully-polarimetric SAR data at L-band $(1.3 \mathrm{GHz})$ and $\mathrm{P}$-band $(0.35 \mathrm{GHz})$ were acquired at the "summit" test site, located in the accumulation zone, by DLR's airborne E-SAR sensor including a South- and a North-heading [8]. Fig. 1 shows a Pauli-RGB representation of the polarimetric data for a first analysis on elementary scattering mechanisms. For L-band mainly a trend over range is observed, which is similar for both headings. Moving from L-band to P-band a similar trend is present for North and South tracks. For both frequencies ice features can be distinguished from the range trend by comparing their location on both headings. However, their intensities differ depending on frequency.

\section{RESULTS}

The developed polarimetric ice volume component was compared to the presented PolSAR data. The polarimetric entropy $(H)$, mean scattering alpha angle $(\alpha)$ [4], copolarization ratio and phase, were derived from the data by averaging along the entire azimuth direction (North $\mathrm{N}$ : Blue line, South S: Red line), and then compared to the modeled results (green line, purple line $=$ no differential propagation effects included). 

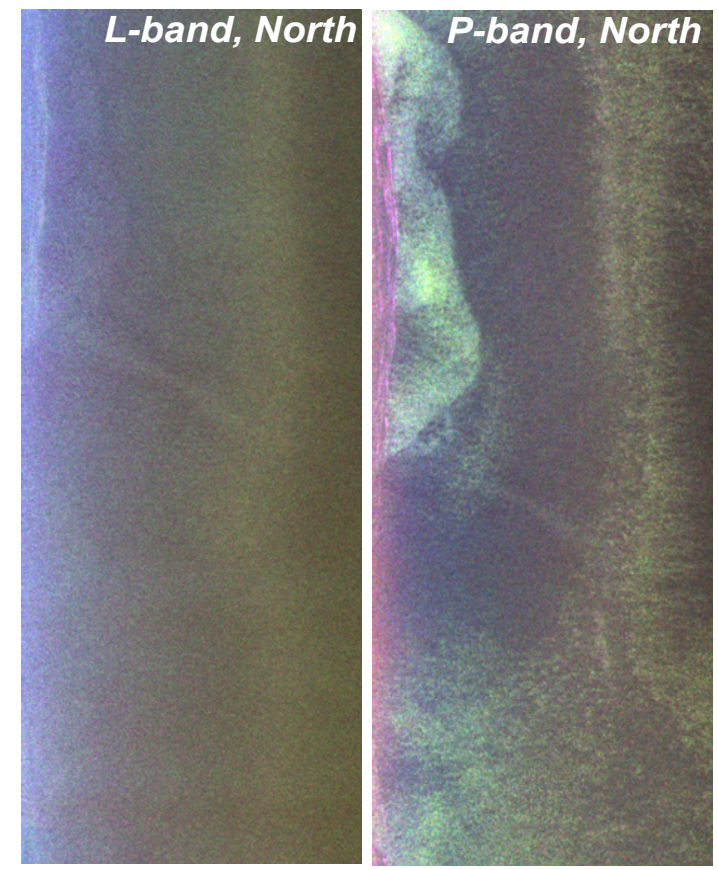

Fig.2: RGB Pauli images (R: $1 / 2<\left|\mathrm{S}_{\mathrm{HH}}-\mathrm{S}_{\mathrm{VV}}\right|>$, G: $2<\left|\mathrm{S}_{\mathrm{XX}}\right|>$, B: $\mathbf{1} / \mathbf{2}<\left|\mathrm{S}_{\mathrm{HH}}+\mathrm{S}_{\mathrm{VV}}\right|>$ ) at $\mathrm{L}$ - and P-band for the North-heading on the "summit" test site; Left edge = near range.

In the case of no differential propagation the co-polarization phase is always zero, whereas the co-polarization ratio is not significantly affected by this phenomenon. For each data profile in Figs. 3-4, standard deviations along azimuth direction are included for certain range positions. Fig. 3 shows the data-to-model comparison when air bubbles $\left(\varepsilon_{r}=\right.$ 1.0) are assumed as inclusions in the ice volume (L-band: $\varepsilon_{r}=3.15$-j0.008, P-band: $\left.\varepsilon_{r}=3.0-\mathrm{j} 0.012\right)$ and the model is set up with $A_{p}=0.3$ (oblate air bubbles) and $\mu_{i}=3.5 \%$. A completely random distribution is assumed for the canting angle, whereas the mean tilt angle is set to $0^{\circ}$ (horizontal oblates) with a gentle distribution width of $60^{\circ}$. At L-band, the model shows a good agreement with the data for all the parameters. The impact of differential propagation effects on $H$ and $\alpha$ can be easily evaluated by comparing the complete volume scattering component (green lines) with the case, which neglects these effects (purple lines). The upper left graph in Fig.3 clearly indicates that this phenomenon can explain the occurring co-polarization phase and its trend along range. Nevertheless, the phase behavior in the very far range is still a matter of ongoing research.

Moving to P-band (Fig. 4), the polarimetric analysis of the data shows a different scenario compared to L-band. Modeled $H$ and $\alpha$ values are still in good agreement with the data, if the deviation for near range $\left(<30^{\circ}\right)$ is omitted. For the co-polarization phase, an anomalous range (from negative to positive values) is observed, despite the range trend (increasingly monotonous) and the dynamics (around $\left.50^{\circ}\right)$ are very similar to the L-band case, and both tracks $(\mathrm{N}$,
S) are in substantial agreement. Finally, the co-polarization ratio shows range trends with significant difference between $\mathrm{N}$ and $\mathrm{S}$. This might be due to underlying ice features, also visible in the Pauli image, and not explainable with range (incidence angle) dependencies. This result might be traced back to the higher penetration depth of P- compared to Lband, for which the polarimetric signatures appear more homogeneous along range.

\section{CONCLUSIONS}

The proposed volume scattering component for glacier ice volumes was compared to experimental PolSAR data at Land P-band. Polarimetric signatures, showing a characteristic range dependency, could be well tracked by the volume scattering component at L-band, because this component includes the variability of the incident angle along range. This explains especially the range trend of the co-polarization ratio. In addition, the co-polarization phase and its behavior along range can be traced, when the differential propagation effects within the glacier volume are incorporated. After inclusion of both (incidence angle, propagation effects), the entropy and scattering alpha angles of the volume component and the data coincide well at Lband. However, the deeper penetration of P-band emphasizes the presence of local ice structures that are not explicitly included in the volume component, since they are not range dependent. This highlights that the developed polarimetric approach works properly, when homogeneous ice volumes are considered, while it needs to be further adapted and extended (by additional components) to incorporate the inhomogeneities appearing with depth.

\section{REFERENCES}

[1] J.J. Sharma, I. Hajnsek, K. Papathanassiou, and A. Moreira, "Polarimetric Decomposition Over Glacier Ice Using Longwavelength Airborne PolSAR", IEEE TGRS, vol. 49, no.1, pp. 519-535, 2011.

[2] A. Freeman and S. L. Durden, "A three-component scattering model for polarimetric SAR data," IEEE TGRS, vol. 36, no. 3, pp. 963-973, May 1998.

[3] W.S.B. Paterson, "The Physics of Glaciers", Butterworth Heinemann, 1994.

[4] S.R. Cloude, "Polarisation: Applications in Remote Sensing", Oxford, 2010.

[5] F.T. Ulaby, R.K. Moore, and A.K. Fung, "Microwave Remote Sensing, Active and Passive, Volume II: Fundamentals and Radiometry", Norwood, MA: Addison-Wesley, 1981.

[6] H.C. van de Hulst, "Light Scattering by Small Particles", Dover, 1981.

[7] S.R. Cloude, K.P. Papathanassiou, W.M. Boerner, "The Remote Sensing of Oriented Volume Scattering Using Polarimetric Radar Interferometry", Proceedings of ISAP, Fukuoka, Japan, pp. 549-552, 2000.

[8] I. Hajnsek, R. Horn, R. Scheiber, J.J. Sharma, "ICESAR 2007: Technical Assistance for the Deployment of Airborne SAR and Geophysical Measurements during the IceSAR 2007," ESA Study Final Report, no. 20655/07/NL/CB, 2008. 

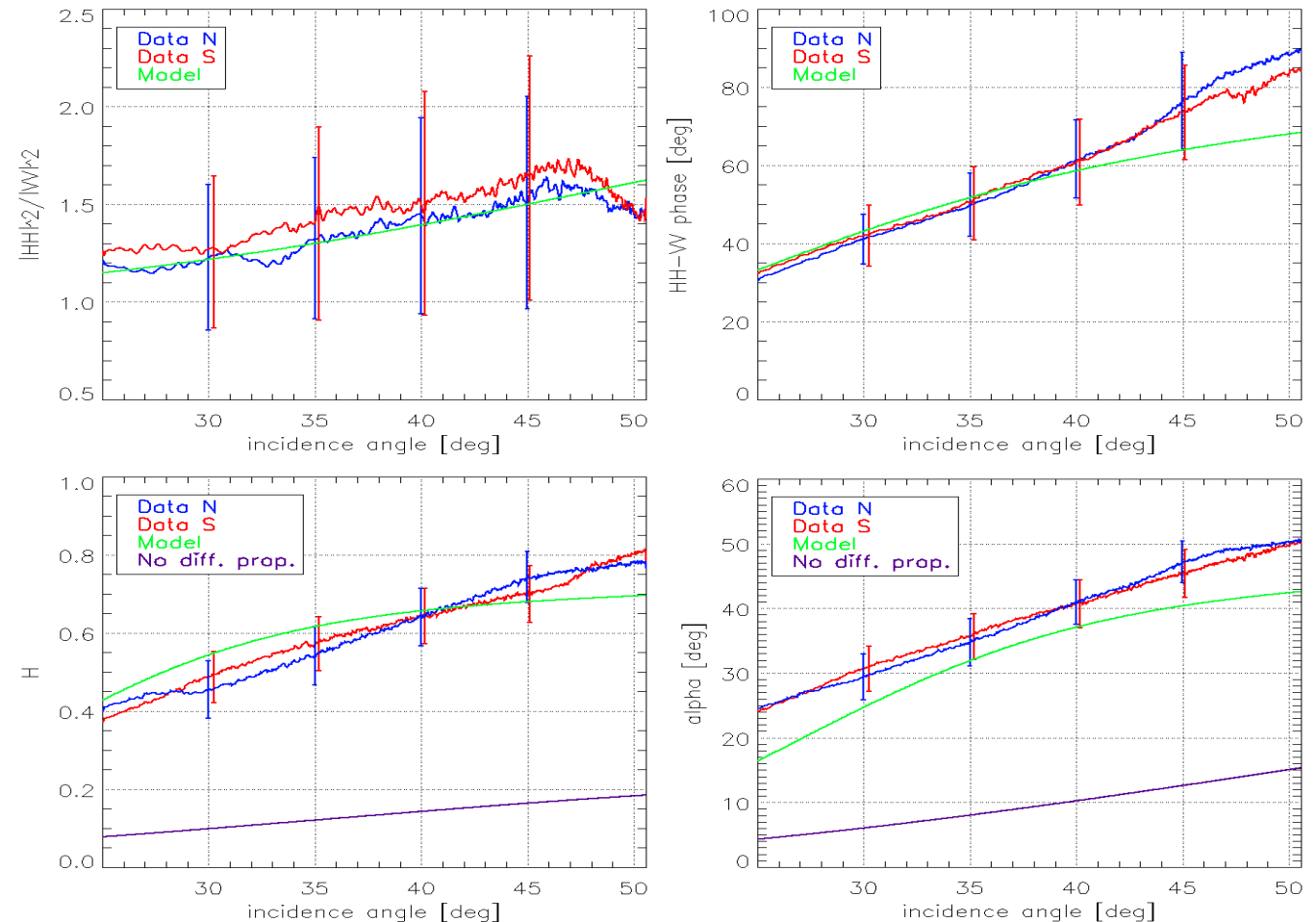

Fig.3: L-band range profile of polarimetric signatures for North (blue) and South (red) headings, compared to the modeled profiles with (green) and without (purple) inclusion of differential propagation effects.
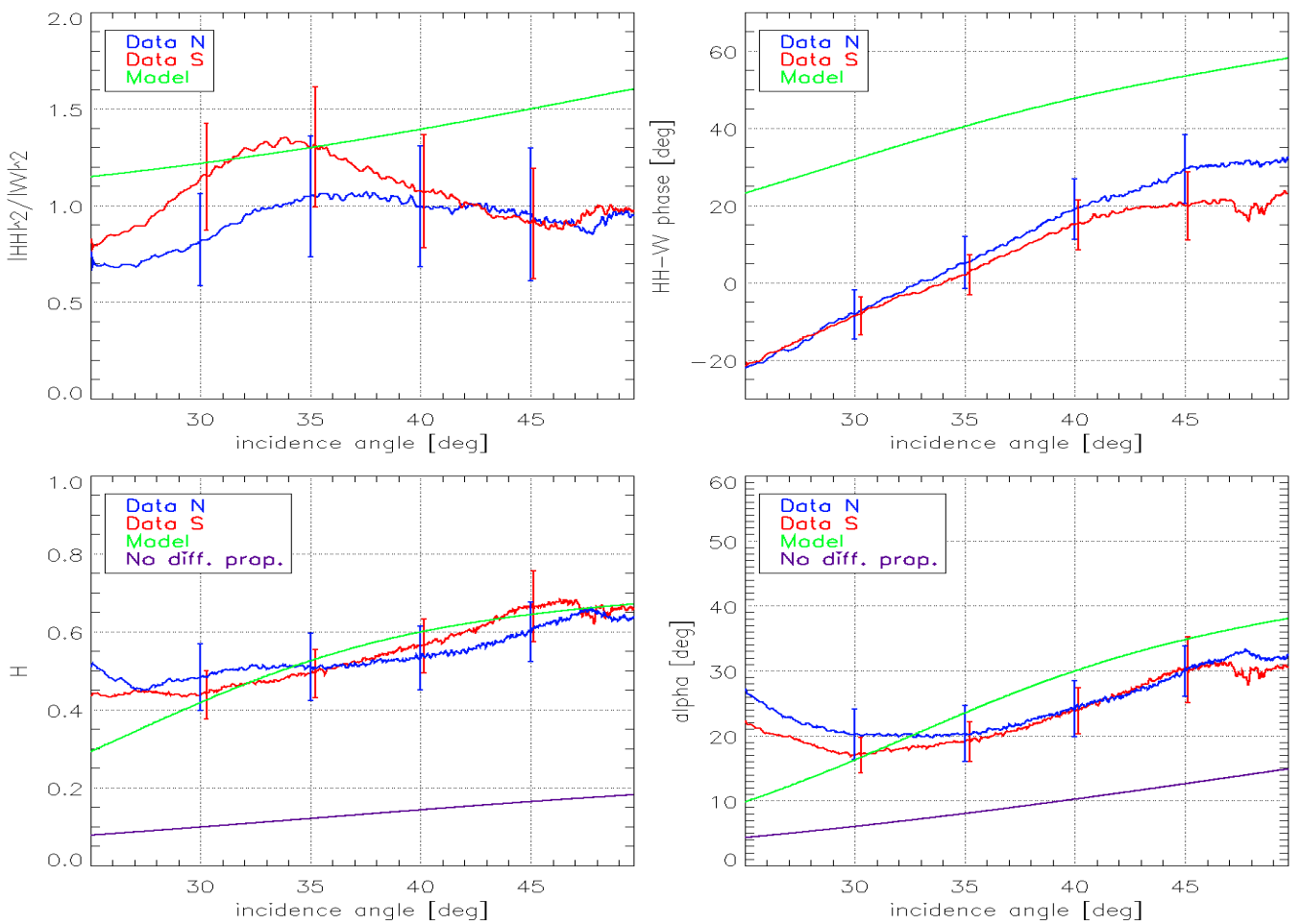

Fig.4: P-band range profile of polarimetric signatures for North (blue) and South (red) headings, compared to the modeled profiles with (green) and without (purple) inclusion of differential propagation effects. 